\title{
Influence of periodontal ligament simulation on bond strength and fracture resistance of roots restored with fiber posts
}

\author{
Ana Maria Estivalete MARCHIONATTI' ${ }^{1}$ Vinícius Felipe WANDSCHER ${ }^{1}$, Juliana BROCH ${ }^{1}$, César Dalmolin BERGOLI², \\ Juliana MAIER ${ }^{3}$, Luiz Felipe VALANDRO', Osvaldo Bazzan KAIZER ${ }^{1}$
}

\footnotetext{
1- Department of Restorative Dentistry, School of Dentistry, Federal University of Santa Maria, Santa Maria, RS, Brazil.

2- Department of Restorative Dentistry, School of Dentistry, Federal University of Pelotas, Pelotas, RS, Brazil.

3- Department of Stomatology, School of Dentistry, Federal University of Santa Maria, Santa Maria, RS, Brazil.
}

Corresponding address: Ana Maria Estivalete Marchionatti - Universidade Federal de Santa Maria - R. Marechal Floriano Peixoto - 1184 - $3^{\circ}$ andar - sala 312 - 97015-372 - Santa Maria - RS - Brazil - Phone: +55 (55) 32209210 - e-mail: anamarchionatti@hotmail.com

Submitted: February 19, 2014 - Modification: June 16, 2014 - Accepted: July 8, 2014

\section{ABSTRACT}

\begin{abstract}
bjective: Considering that periodontal ligament simulation may influence the stress distribution over teeth restored with intraradicular retainers, this study aimed to assess the combined effect of mechanical cycling and periodontal ligament simulation on both the bond strength between fiber posts and root dentin and the fracture resistance of teeth restored using glass fiber posts. Material and Methods: Ninety roots were randomly distributed into 3 groups $(n=10)$ (C-MC: control; P-MC: polyether; AS-MC: addition silicone) to test bond strength and 6 groups $(n=10)$ (C: control; P: polyether; AS: addition silicone, without mechanical cycling, and C-MC, P-MC and AS-MC with mechanical cycling) to test fracture strength, according to the material used to simulate the periodontal ligament. For the bond strength test, fiber posts were cemented, cores were built, mechanical cycling was applied $\left(2 \times 10^{6}\right.$ cycles, $88 \mathrm{~N}, 2.2 \mathrm{~Hz}$, and $45^{\circ}$ incline), and the teeth cut into 3 slices $(2 \mathrm{~mm})$, which were then subjected to the push-out test at $1 \mathrm{~mm} / \mathrm{min}$. For the fracture strength test, fiber posts were cemented, cores were built, and half of the groups received mechanical cycling, followed by the compressive strength $\left(45^{\circ}\right.$ to the long axis and $1 \mathrm{~mm} / \mathrm{min}$ ) performed on all groups. Results: Periodontal ligament simulation did not affect the bond strength $(p=0.244)$ between post and dentin. Simulation of periodontal ligament $(p=0.153)$ and application of mechanical cycling $(p=0.97)$ did not affect fracture resistance. Conclusions: The materials used to simulate the periodontal ligament did not affect fracture or bond strength, therefore periodontal ligament simulation using the tested materials could be considered optional in the conditions of the study.
\end{abstract}

Keywords: Dental pins. Fatigue. Compressive strength. Dental bonding.

\section{INTRODUCTION}

For the reconstruction of endodontically treated teeth, intraradicular posts are recommended to retain the restoration when there is less than $50 \%$ of the coronal structure remaining ${ }^{21}$. Among other intraradicular anchorage options, adhesively cemented fiber posts reduce the risk of root fracture because they have an elastic modulus $(E=40$ $\mathrm{GPa})$ similar to dentin $(\mathrm{E}=18 \mathrm{GPa})$, unlike ceramic $(E=200 \mathrm{GPa})$ and metallic posts $(E=200 \mathrm{GPa})^{6,17}$.
Consequently, fiber posts provide more uniform stress distribution on root dentin compared to other retainers ${ }^{6,13}$.

The clinical success of restorative treatments depends on many factors, including the amount of dental substrate ${ }^{7}$, adequate coronal reconstruction ${ }^{7}$, cavity configuration factor ${ }^{19}$, masticatory load support by the teeth and stress distribution over the bone and periodontal ligament ${ }^{26}$. The periodontal ligament, ranging between 0.1 and $0.3 \mathrm{~mm}$ thick ${ }^{16}$, absorbs occlusal loads; the periodontal fiber compression 
provides important support when the tooth is submitted to external forces ${ }^{24}$. When a force is applied, the resistance of the periodontal membrane is initially low, but as the load increases, the resistance also increases gradually until tooth dislodgment becomes minimal. The periodontal ligament is compressive to a limited extent and partially elastic ${ }^{24,26}$. For masticatory loads up to $70 \mathrm{~N}$, the periodontal ligament compresses to approximately 0.03-0.15 $\mathrm{mm}^{25}$. When the fibers reach their maximum load resistance, the periodontal ligament becomes rigid, the load is transmitted to the bone, and tensions are distributed over the root surface ${ }^{26}$.

Therefore, the properties of masticatory force absorption through the bone and periodontal ligament should be reproduced in laboratorial tests in order to simulate the clinical reality more accurately ${ }^{26}$. However, various studies that assess bond strength between root canal posts and dentin after mechanical fatigue do not simulate periodontal ligament ${ }^{3,8}$. Similarly, most studies fail to simulate the periodontal ligament in fracture resistance tests ${ }^{5,18}$, although a few studies simulate the periodontal ligament using polyether ${ }^{11,13}$ and polivynilsiloxanebased elastomers ${ }^{7,22}$.

A few in vitro studies assessed the effects of periodontal ligament simulation and showed that an artificial periodontal ligament reduced the fracture strength and modified the failure modes of sound in bovine teeth ${ }^{26}$. Also, simulating the periodontal ligament significantly reduced fracture resistance of ceramic-fixed partial dentures ${ }^{25}$.

Considering that root posts reduce the area available for stress distribution, concentrating it on the remaining dental structure and the dentinpost interface ${ }^{14}$, periodontal ligament simulation may interfere in stress distribution. Therefore, the objectives of the present study were to characterize the influence of different artificial periodontal ligament materials on fiber post-root dentin bond strength after mechanical cycling, and to evaluate the effect of periodontal ligament simulation on fracture resistance of teeth restored with glass fiber posts, both with and without simultaneous mechanical cycling. Two hypotheses were tested: 1) a simulated periodontal ligament under mechanical cycling would not affect bond strength, and 2) periodontal ligament simulation would not affect fracture resistance.

\section{MATERIAL AND METHODS}

\section{Specimen selection and adequacy}

Ninety single-root bovine teeth were cleansed with periodontal curettes and stored in distilled water. Coronal portions were sectioned under water cooling to obtain a standardized $16 \mathrm{~mm}$ root length. The most coronal diameter of the root canal was measured with a digital caliper (Starrett 727, Starret, Itu, SP, Brazil), and any tooth with a canal diameter larger than the fiber post diameter $(\Phi=1.8 \mathrm{~mm}$, White Post \#3, FGM, Joinville, SC, Brazil) was discarded and replaced with a different specimen.

The apical foramens were sealed using a photo activated adhesive (Single Bond 2, 3M ESPE, Saint Paul, MN, USA) associated with a composite resin (Oppalis, FGM). The canals were prepared with the same drill used in the posts system (White Post DC \#3, FGM) to a $12 \mathrm{~mm}$ length.

\section{Experimental design: root embedment and periodontal ligament simulation}

The roots were numbered from 1 to 90 and randomly distributed into 6 groups $(n=15)$, which varied by the artificial periodontal ligament composition and the test performed (Figure 1). The number sequences were generated by Random Allocation Software (developed by M. Saghaei, Isfahan University of Medical Sciences, Isfahan, Iran).

The surfaces of 60 roots were covered with baseplate wax (Lysanda, São Paulo, SP, Brazil) that was liquefied at $60^{\circ} \mathrm{C}$. To simulate the biological space, each root was immersed in wax for 2 seconds up to $3 \mathrm{~mm}$ below the most coronal portion of the root, which created an approximately $0.3 \mathrm{~mm}$ thick wax layer, considered adequate in prior literature ${ }^{10,26}$. The thickness of the wax layer was confirmed by measuring the thickness of the root with a digital caliper before and after immersion.

\begin{tabular}{|c|c|c|c|}
\hline & & \multicolumn{2}{|c|}{ Groups } \\
\hline Test & $\begin{array}{c}\text { Periodontal ligament } \\
\text { simulation }\end{array}$ & Mechanical cycling (MC) \\
\hline Fracture resistance & Control & No \\
& Polyether & C-MC & P \\
& Addition silicone & AS-MC & AS \\
\hline Push out bond strength & & & - Control \\
& Polyether & P-MC & AS-MC \\
\hline
\end{tabular}

Figure 1- Experimental design 
All 90 roots were embedded in PVC cylinders (height: $14 \mathrm{~mm}$; diameter: $25 \mathrm{~mm}$ ) as described in other study ${ }^{4}$. The bur used to prepare the conduits was positioned inside the canal, and while supporting the root, attached to a surveyor. Consequently, the long axes of the bur, the root, and the cylinder were parallel to each other and perpendicular to the horizontal axis. Self-curing acrylic resin Dencrilay (Dencril, Caieiras, SP, Brazil) was mixed at $3: 1$ (powder:liquid) and poured inside the PVC cylinder. The most coronal $3 \mathrm{~mm}$ section of the root was exposed to simulate the bone limit.

After resin polymerization, the teeth covered bywax were separated from the cylinders, and the wax was removed from the root surface and acrylic resin interior using hot water and manual instruments.

Thirty roots had periodontal ligament simulated with polyether (Impregum Soft Medium Body, $3 M$ ESPE) and 30 roots with addition silicone (Express Medium Body, 3M ESPE), according to the randomization. To create the artificial periodontal ligament, the corresponding elastomeric material was inserted along the artificial alveolus created on the acrylic resin. Then, the root was repositioned into the alveolar space and the elastomer polymerized; the elastomer in excess was then removed using a scalpel blade.

\section{Posts cementation}

The glass fiber posts were sectioned to a length of $17 \mathrm{~mm}$ ( $5 \mathrm{~mm}$ coronal portion and $12 \mathrm{~mm}$ intracanal portion). Each post was cleansed with $70 \%$ ethanol; silane coupling agent (Prosil, FGM) was applied onto the surface using a microbrush (Cavibrush, FGM), and the posts were left undisturbed for 5 minutes, according to the recommendations of the manufacturer.

The root canals were conditioned with $37 \%$ phosphoric acid (Condac 37, FGM) for 15 seconds and washed with $10 \mathrm{~mL}$ of distilled water. Excess water was removed with absorbent paper points (Tanari, Manacapuru, AM, Brazil). The activator of the adhesive system (Adper Scotchbond Multipurpose, $3 \mathrm{M}$ ESPE) was applied into the canal using a microbrush (Cavibrush, FGM); the excess material was removed with paper points (Tanari) and put to dry for 5 seconds. The adhesive system Primer and Catalyst were applied in a similar manner. Then, the resin cement RelyX ARC (3M ESPE) was applied on the post with a spatula and in the root canal with a Lentulo drill \#40 (Injecta, Diadema, SP, Brazil). The posts were positioned inside the root canal, the cement in excess was removed, and photo-activation was performed for 40 seconds on the occlusal surface with a LED (Radii-Cal, SDI, Melbourne, Vic, Australia).

After the posts were cemented, the crown was reconstructed. The coronal dentin was etched with $37 \%$ phosphoric acid (Condac 37, FGM) for 15 seconds, washed with $10 \mathrm{~mL}$ of distilled water, and the excess removed with absorbent paper. The adhesive system (Single Bond 2, 3M ESPE) was applied using a microbrush (Cavibrush, FGM); the core was built up with composite resin (Oppallis, FGM) using matrices at standardized dimensions and anatomical configuration of an upper central incisor prepared for a full crown. The specimens were stored in distilled water at $37^{\circ} \mathrm{C}$ for 24 hours.

\section{Push-out bond strength test}

The groups $(n=10)$ were divided according to the artificial periodontal ligament composition (Figure 1 ). All the specimens were immersed in distilled water at $37^{\circ} \mathrm{C}$ and submitted to $2 \times 10^{6}$ cycles in a mechanical fatigue simulator (ER 11000, Erios, São Paulo, SP, Brazil), which is equivalent to 2 years of clinical service ${ }^{30}$ at an $88 \mathrm{~N}$ load, $2.2 \mathrm{~Hz}$ frequency and $45^{\circ}$ incline from the horizontal axis. Twenty-four hours after the mechanical cycling was finished, the specimens were fixed on the metallic base of a cutting machine (LabCut 1010, Extec Corp., Enfield, CT, USA) and sectioned perpendicularly to the root long axis. The first slice of approximately $1 \mathrm{~mm}$ was discarded, and 3 slices ( $2 \mathrm{~mm}$ ) were obtained from each tooth. Each slice was positioned with the most coronal portion downward on a metallic device while a cylinder applied a load on the post directed from the apical to the coronal portion at a crosshead speed of $1 \mathrm{~mm} / \mathrm{min}$ (Emic DL 2000, Emic, São José dos Pinhais, PR, Brazil).

The height and canal diameters of the coronal and apical portions were measured with a digital caliper. The bond strength ( $\mathrm{MPa}$ ) was calculated using the formula $R=F / A$, where $F$ was the force of rupture $(N)$, and $A$ was the bonded area. The bonded area was calculated using the formula to determine the lateral area of a circular straight cone with parallel bases: $A=\pi g .(R 1+R 2)$, where $\pi=3.14, g=$ slant height, $\mathrm{R} 1=$ smaller base radius, and $\mathrm{R} 2=$ larger base radius. To determine the slant height, the following formula was used: $g^{2}=h^{2}+(R 2-R 1)^{2}$, where $h=$ specimen thickness and R1 and R2 were the internal diameters of the smaller and larger bases, respectively, divided by 2 .

Afterwards, the specimens were examined under a Stereo Discovery V20 optical microscope (Carl Zeiss, Göttingen, NSAC, Germany) up to $40 \times$ to determine the predominant failure pattern. Failures were classified as: adhesive failure between dentin and cement (Ad DC); adhesive failure between cement and post (Ad CP); cohesive failure of the dentin (Co $D)$; cohesive failure of the cement (Co C); cohesive failure of the post ( $C o P$ ); mixed adhesive failure of dentin/adhesive failure between post and cement (M Ad DC/PC); and mixed adhesive/cohesive failure (M Ad/Co). 
Specimens with representative failures were analyzed using scanning electron microscopy (JEOLJSM-5400, Jeol, Tachikawa, TY, Japan).

\section{Fracture resistance test}

The groups $(n=10)$ were divided according to the periodontal ligament composition and application of mechanical cycling (Figure 1). Specimens of groups C-MC, P-MC and AS-MC were submitted to mechanical cycling according to the same protocol
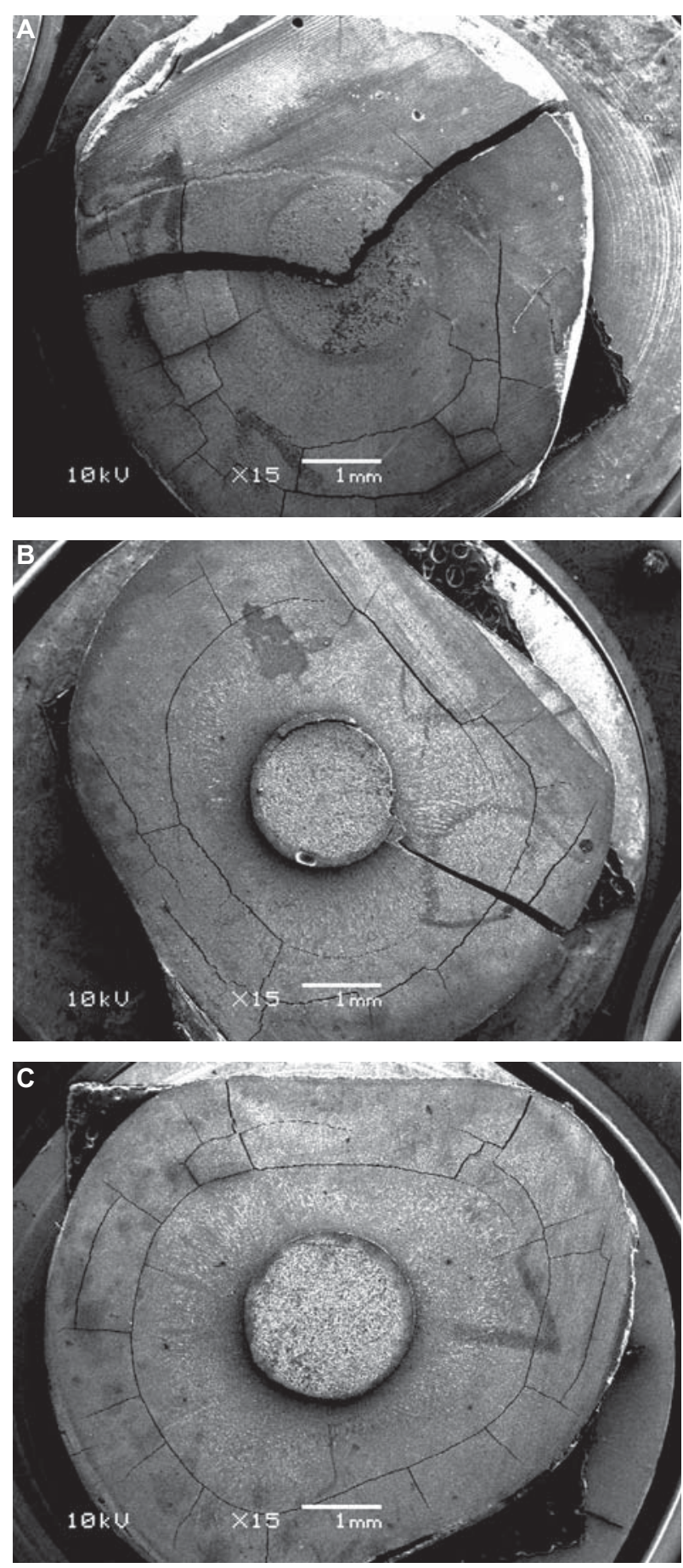

Figure 2- Scanning electron microscopy images. (A) Cohesive failure in dentine. (B) Mixed adhesive/cohesive failure. (C) Adhesive failure between dentin and cement used for the push-out test. The specimens that were not mechanically cycled were stored in distilled water at a temperature of $37^{\circ} \mathrm{C}$ during the same time period of the mechanical cycling.

Twenty-four hours after the mechanical cycling was finished, all specimens of each group were submitted to a compressive strength test in a universal testing machine (Emic DL 2000, Emic). Each specimen was positioned on the device aligned at $45^{\circ}$ to the tooth long axis, and a constant static load was applied at a crosshead speed of $1 \mathrm{~mm} / \mathrm{min}$ until failure. Failure was defined as the point when the load $(\mathrm{N})$ reached its maximum value during the test.

The failure modes were classified as favorable and unfavorable under an optical microscope (Stereo Discovery V20, Carl Zeiss). Fractures at or above the simulated bone level, fractures of the coronal portion of the core, and dislodgement of the core and/or post coronal portion were considered favorable failures. Vertical or oblique radicular fractures below the simulated bone level were classified as unfavorable failures.

\section{Statistical analysis}

Normal distribution and homoscedasticity were verified with Shapiro-Wilk and Levene tests. Bond strength values were analyzed by one-way ANOVA, with periodontal ligament material as the variable, and fracture strength values were analyzed by two-way ANOVA, with the periodontal ligament composition and application of mechanical cycling as variables. In both cases, the significance level was $5 \%$.

Cohesive (Figure $2 \mathrm{~A}$ ) or mixed adhesive/cohesive (Figure 2B) failure slices were excluded from the bond strength statistical analysis because they did not represent adhesive failures.

\section{RESULTS}

There was no root fracture or restoration failure during the mechanical cycling.

\section{Push-out bond strength test}

Table 1 summarizes the bond strength means and standard deviations. One-way ANOVA revealed that the periodontal ligament simulation did not affect the bond strength $(p=0.244)$. Most of the failures were

Table 1- Push-out bond strength (MPa) means \pm standard deviations

\begin{tabular}{cc}
\hline Group $^{*}$ & Mean \pm SD \\
\hline C & $10.82 \pm 2.93$ \\
P & $13.06 \pm 2.38$ \\
AS & $11.07 \pm 3.23$ \\
\hline
\end{tabular}

${ }^{*}$ C: Control; P: Polyether; AS: Addition Silicone 
Table 2- Distribution of failure modes after the push-out test

\begin{tabular}{|c|c|c|c|c|c|c|c|}
\hline \multirow[b]{2}{*}{ Group* } & \multicolumn{7}{|c|}{ Failure mode** } \\
\hline & Ad DC & Ad CP & Co D & Co C & Co $P$ & M Ad DC/PC & M Ad/Co \\
\hline C-MC & 35 & 1 & 2 & - & - & 1 & - \\
\hline P-MC & 30 & 6 & - & - & - & 1 & 2 \\
\hline AS-MC & 31 & 4 & - & - & - & 3 & - \\
\hline Total & $96(83 \%)$ & $11(9 \%)$ & $2(2 \%)$ & 0 & 0 & $5(4 \%)$ & $2(2 \%)$ \\
\hline
\end{tabular}

*All groups were submitted to mechanical cycling. ${ }^{* *} \mathrm{Ad} \mathrm{DC}$ : adhesive failure between dentin and resin cement; Ad CP: adhesive failure between dentin and fiber post; Co D: cohesive failure of dentin; Co P: cohesive failure of fiber post; $\mathrm{M}$ Ad DC/PC: mixed failure with adhesive failure between dentin/resin cement and fiber post/resin cement; M Ad/Co: mixed failure with adhesive and cohesive failures

Table 3- Means \pm standard deviation of the fracture load $(\mathrm{N})$ data

\begin{tabular}{ccc}
\hline Group* $^{*}$ & Mechanical cycling & Mean \pm SD \\
\hline CP & No & $415.20 \pm 121.70$ \\
P & & $327.16 \pm 51.40$ \\
AS & & $367.18 \pm 94.27$ \\
\hline C-MC & Yes & $381.76 \pm 110.20$ \\
P-MC & & $346.69 \pm 51.08$ \\
AS-MC & & $378.21 \pm 134.35$ \\
\hline
\end{tabular}

${ }^{*} \mathrm{C}, \mathrm{P}$ and AS were not mechanically cycled; C: control; P: polyether; AS: addition silicone; C-MC: control submitted to mechanical cycling; P-MC: polyether submitted to mechanical cycling; AS-MC: addition silicone submitted to mechanical cycling

Table 4- Qualitative analysis of failure patterns after the fracture resistance test

\begin{tabular}{|c|c|c|c|c|c|c|c|c|c|}
\hline & \multicolumn{9}{|c|}{ Groups } \\
\hline & & \multicolumn{3}{|c|}{ Without mechanical cycling } & \multicolumn{3}{|c|}{ With mechanical cycling } & \multirow[b]{2}{*}{ Total } & \multirow[b]{2}{*}{$\%$} \\
\hline & & C & $\mathbf{P}$ & AS & C-MC & P-MC & AS-MC & & \\
\hline \multirow{2}{*}{$\begin{array}{l}\text { Failure } \\
\text { pattern }\end{array}$} & Favorable & 6 & 8 & 9 & 10 & 10 & 10 & 53 & 88 \\
\hline & Unfavorable & 4 & 2 & 1 & - & - & - & 7 & 12 \\
\hline \multirow[t]{3}{*}{ Failure place } & Core fracture & 9 & 8 & 9 & 10 & 10 & 10 & 56 & \\
\hline & Post fracture* & - & - & - & - & - & - & & \\
\hline & Root fracture & 4 & 2 & 1 & - & - & - & 7 & \\
\hline \multirow{2}{*}{$\begin{array}{l}\text { Failure } \\
\text { direction }\end{array}$} & Mesiodistal & 10 & 10 & 10 & 10 & 7 & 9 & 56 & 93 \\
\hline & Buccolingual & - & - & - & - & 3 & 1 & 4 & 7 \\
\hline
\end{tabular}

* No post fracture was observed, however, a mesiodistal crack was observed in the posts of several specimens (Figure 4) C: control; P: polyether; AS: addition silicone

adhesive and occurred between the dentin and resin cement (Figure 2C), followed by adhesive failures between the post and resin cement (Table 2).

\section{Fracture resistance test}

Means and standard deviations are presented in Table 3. Two-way ANOVA showed that the simulation of the periodontal ligament $(p=0.153)$, the mechanical cycling ( $p=0.97)$, and the interaction between the two factors $(p=0.665)$ did not significantly affect fracture resistance. Favorable failures were predominant among other failure modes (Table 4).

\section{DISCUSSION}

Periodontal ligament simulation may enable a more accurate in vitro reproduction of the oral environment ${ }^{26}$. In the present study, polyether and addition silicone artificially reproduced the periodontal ligament during mechanical fatigue, after 
the bond strength between the post and dentin was assessed, as well as the fracture resistance of bovine teeth restored using glass fiber posts, both with and without mechanical cycling.

Due to the difficulty of obtaining intact singlerooted human teeth and the ethical aspects implicated in their use for research, bovine teeth were used as substitutes for human teeth. Bovine and human teeth have macro and microscopic structural similarities, and for that reason bovine teeth have been suggested as a possible alternative ${ }^{12}$. Previous laboratorial studies also used bovine teeth as a substitute for human teeth ${ }^{3-5,8,18}$.

The results of the present study showed similar bond strength among the three groups during the push-out test, and thus, the first null hypothesis was accepted. The intraradicular retainers used in the current study were glass fiber posts, which, based on finite element analysis, generated stress distribution on the root similar to non-restored teeth, as a consequence of its elastic modulus ${ }^{14,17}$. Based on those studies, the lack of differences between the tested groups may result from a tendency towards homogeneous force distribution during mechanical cycling, reducing stress applied on the post/dentin interface so that the load absorbed by the periodontal ligament may not have been relevant in the study conditions. By contrast, when metallic or ceramic intraradicular posts are used, concentrated tension zones are created because the reconstruction material is more rigid than dentin ${ }^{6,17}$. The present results may have been different if metallic or ceramic posts were used instead.

In endodontically treated teeth restored with posts, shear forces are present in the central region of the specimens, which can lead to adhesive failure at the dentin/post interface ${ }^{29}$. Although the finding was not statistically significant, the group lacking an artificial periodontal ligament simulation had lower bond strength than the other groups. If a higher number of cycles had been applied on the specimens during aging, perhaps a significant reduction of the bond strength could have been induced.

The push-out test generated predominantly adhesive failures at the dentin/cement interface, similarly to previous studies $3,8,9,15,19$. That failure pattern is probably due to the high $\mathrm{C}$ factor inside the canal and the difficulty visualizing and controlling the moisture of the radicular dentin ${ }^{15,19}$. Furthermore, 9\% of adhesive failures between cement and post may have been caused by the strong adhesion between dentin and cement promoted by the three-step totaletch adhesive system ${ }^{3}$.

The second null hypothesis was also accepted because there was no statistical difference between the groups in fracture resistance regardless of mechanical cycling. This result contrasts with those of a previous study ${ }^{26}$ that observed a significant difference between the groups with and without a periodontal ligament after fracture strength testing; however, the study did not perform mechanical cycling, and intact bovine teeth were used, which have longer and more voluminous crowns than the specimens utilized in the present study.

Bearing in mind the aforementioned finite elements analysis studies ${ }^{14,17}$, this finding may be associated with the primarily uniform stress distribution on teeth restored with glass fiber posts. When fiber posts are used, stress concentration is lower compared to rigid retainers ${ }^{6}$, and thus, the cushioning effect provided by the impression material did not alter fracture resistance. However, if metallic or ceramic posts are used, high stress concentration

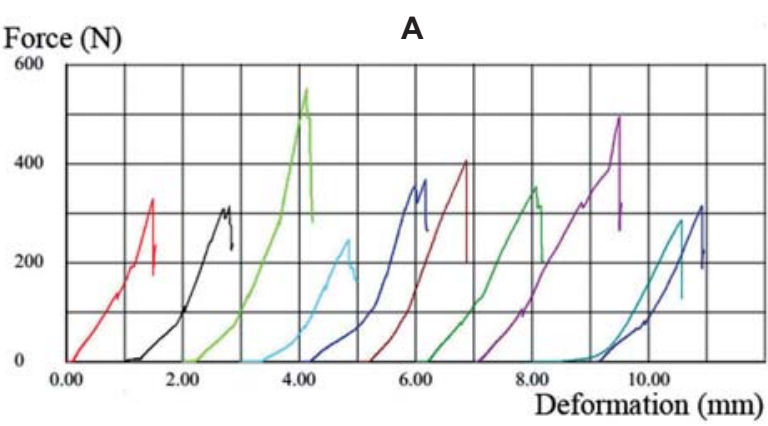

Force (N)

B

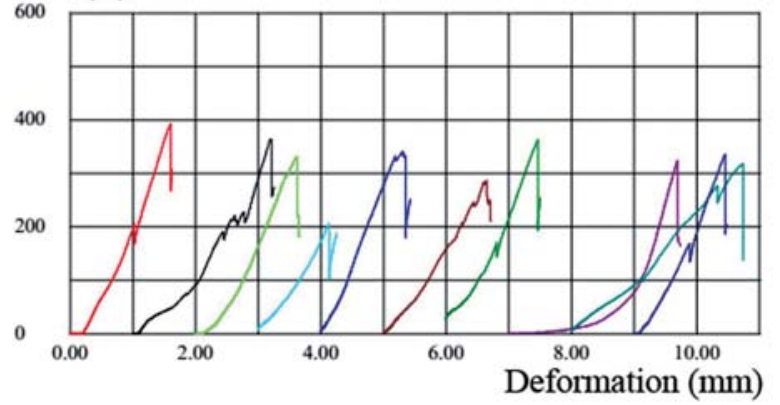

Force $(\mathrm{N})$

C

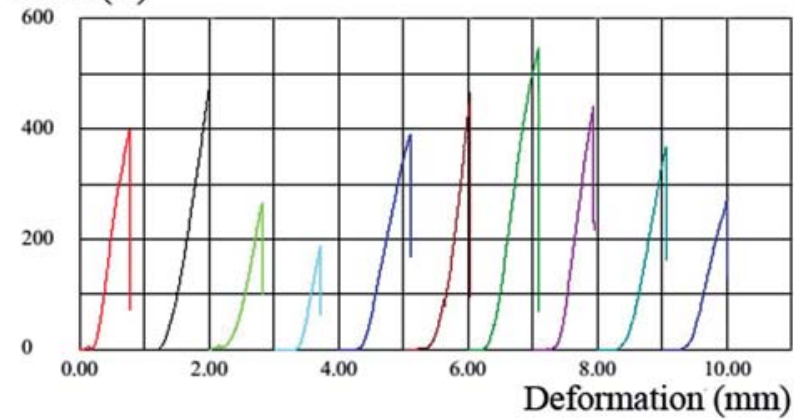

Figure 3- Graphics of groups addition silicone $(A)$, polyether $(\mathrm{B})$, and control $(\mathrm{C})$ illustrating the force $(\mathrm{N})$ and deformation $(\mathrm{mm})$ of each specimen during the fracture strength test. In the groups with an artificial periodontal ligament, the specimens are accommodated when a load is applied. This is not observed in group $C$ because of the rigidity of the acrylic resin, and the graphic is a crescent line until the failure threshold of the restorative assembly is reached 
is generated on the root portion due to the rigidity of the material 6 . Although the present study did not evaluate those intraradicular retainers, it is speculated that the periodontal ligament simulation may modify stress distribution during mechanical cycling and fracture resistance test if rigid posts are used. More studies must be performed investigating the influence of periodontal ligament simulation on fracture strength of teeth restored with rigid posts and also comparing different post systems.

The absence of difference between experimental and control groups may also be, in part, due to the design of the fracture resistance test. During the test, a load is increasingly applied by an indenter until the restoration fails, which compresses the artificial periodontal ligament between two solid structures. As the elastomer is compressed beyond its elastic limit, its cushioning function is lost ${ }^{1}$, and the load applied by the piston only has effect on the specimen after the elastomer is completely "squeezed" between the specimen and the acrylic resin.

Figure 3 shows the relation between the force applied and the deformation through graphics of fracture resistance tests for groups AS, $P$, and C, generated by the universal testing machine. Graphics of groups AS (Figure 3A) and P (Figure 3B) present premature peaks before failure threshold, which do not indicate fracture, but an accommodation of the specimen in the artificial alveolus by the simulated periodontal ligament. This phenomenon does not occur in the specimens of the group C (Figure 3C), where the only peak illustrated is specimen fracture. Therefore, studies of fracture resistance tests simulating periodontal ligament require attention from the operator to obtain the real value of the fracture.

Mechanical cycling did not affect fracture resistance in any group. Presumably, the protocol used to mechanically cycle the specimens was not sufficient to decrease fracture resistance. These results are in accordance with other studies ${ }^{18,22}$ that also did not find a difference in the fracture resistance of teeth restored with fiber posts before and after mechanical aging.

The failures occurring in the fracture strength test were mainly favorable $(88 \%)$ and likely related to the glass fiber posts and composite resin, which have rigidity similar to that of dentin and provide a relatively uniform stress distribution to the root $^{6}$. In addition, the adhesion between the post and restorative materials (adhesive, cement and composite resin) of similar properties tends to generate a homogeneous stress distribution ${ }^{17}$. Other studies performed in similar conditions also found mostly favorable failures 5,13,22,29.

Failures were predominantly in the core in a mesiodistal direction, which can be explained by the loading applied in a $45^{\circ}$ angle to the palatal surface.
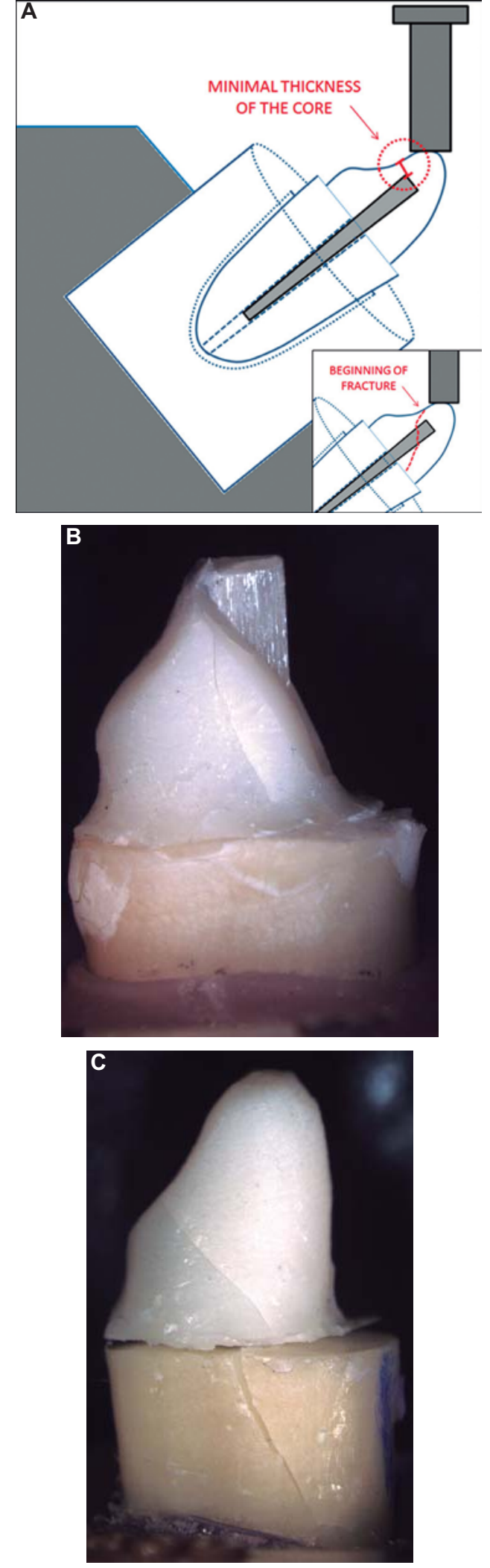

Figure 4- (A) Schematic diagram of the specimen positioned for the test, indicating the minimal thickness area (lingual portion) of the core where the fracture initiates. (B) Stereomicroscope representative image of specimen fractured in the mesiodistal direction (group C). (C) Root fracture associated with core fracture (group addition silicone) after the fracture resistance test 
The specimens bend and experience tensile, and compressive stresses that are maximal externally and minimum centrally. Shear stress also acts on the specimens; howewer, it is maximal in the center and minimal in the external portions ${ }^{29}$. Coronal failures were fractures occurring in the buccal region of the cores with post exposition mesiodistally because of the interaction of tensile, compressive and shear forces. The coronal reconstruction presents a minimal thickness zone of composite resin (Figure 4A), and therefore, the failure tends to initiate in that area (Figure 5A) where tensile stresses are more evident. After failure due to tensile stress, the buccal surface is displaced as a result of the compressive and shear stresses acting on the composite/post interface, and hence, the post becomes exposed (Figure 4B and Figure $5 B$ ). Mesiodistal root fractures are also explained by the same failure mechanism (Figure 4C). The beginning and propagation of the fracture are shown in Figure $5 B$ and $5 C$, respectively.

For the control group without mechanical cycling, there was a higher number of unfavorable failures than in the other groups. When the roots are embedded directly in acrylic resin, there seems to be a stress concentration on the cervical region of the tooth. On the other side, when periodontal ligament simulation is performed, the stress distribution is altered and, instead of occurring stress concentration on a specific region, tension is distributed along the entire root surface ${ }^{26}$.

In the present study, polyether and addition silicone were used to reproduce the periodontal ligament, consistent with other studies performing simulation and using similar elastomeric materials ${ }^{10,11,26,27}$. However, current literature lacks a standardized protocol, and as a result, a wide range of materials, such as condensation silicone ${ }^{23}$, addition silicone ${ }^{7,22}$, polysulfide ${ }^{26}$, polyurethane ${ }^{26,28}$ and an industrial rubber material ${ }^{20}$ have been used, as well as other consistencies of the elastomers used in the present study ${ }^{1,26,27}$. Furthermore, several studies did not indicate the viscosity of the material used $10,13,25$, while others failed to clearly identify the material comprising the artificial periodontal ligament ${ }^{2}$. Additional studies assessing the use of diverse materials and different consistencies are recommended.

Bearing in mind the limitations of the present study, the teeth were restored with composite resin cores instead of crowns, which are not consistent with clinical performance; however, without core protection, the condition generated by load application in the adhesive interface is more hostile $^{8}$, which is an interesting aspect since the present study assessed the bond strength. Also, survival studies, investigation under a longer period of mechanical cycling and finite element analysis could be interesting options for future studies to assess the long-term behavior of endodontically treated teeth restored with intraradicular posts using different simulated periodontal ligaments. Studies evaluating other types of posts are also recommended to validate the influence of an artificial periodontal
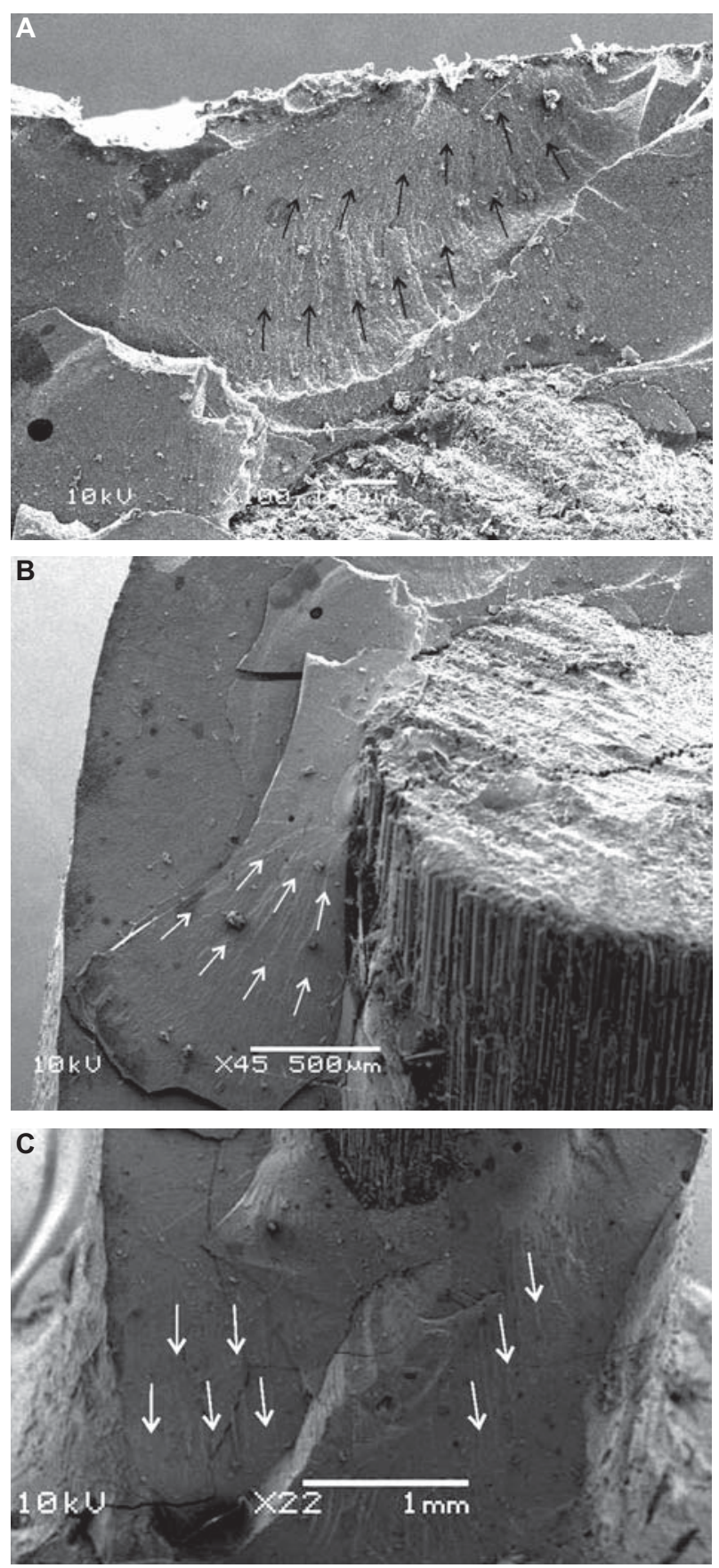

Figure 5- Scanning electron microscopy image of control group. There is displacement of the buccal surface of the core, exposing the post. The fiber post suffers a crack in the mesiodistal direction, which is a consequence of shear stress above the post. (A) Minimal thickness area and hackle lines (black arrows) indicating the start of the fracture. (B) Hackle lines (white arrows) initiating at the composite/post interface. (C) Hackle lines (white arrows) indicated in the buccal surface showing the fracture propagation 
ligament on the behavior of endodontically treated teeth.

\section{CONCLUSION}

The simulation of the periodontal ligament using elastomeric materials does not affect the bond strength and the fracture resistance of the teeth restored with fiber posts and composite cores, and therefore it may not be necessary to perform periodontal ligament simulation in those tests in the experimental conditions of the study.

\section{REFERENCES}

1- Aboushelib MN. Simulation of cumulative damage associated with long term cyclic loading using a multi-level strain accommodating loading protocol. Dent Mater. 2013;29:252-8.

2- Aggarwal V, Singla M, Miglani S, Kohli S. Comparative evaluation of fracture resistance of structurally compromised canals restored with different dowel methods. J Prosthodont. 2012;21:312-6.

3- Amaral M, Rippe MP, Bergoli CD, Monaco C, Valandro LF. Multistep adhesive cementation versus one-step adhesive cementation: push-out bond strength between fiber post and root dentin before and after mechanical cycling. Gen Dent. 2011;59:e185-91.

4- Amaral M, Santini MF, Wandscher V, Amaral R, Valandro LF. An in vitro comparison of different cementation strategies on the pull-out strength of a glass fiber post. Oper Dent. 2009;34:443-51.

5- Amaral M, Santini MF, Wandscher V, Zogheib LV, Valandro LF. Effect of coronal macroretentions and diameter of a glass-FRC on fracture resistance of bovine teeth restored with fiber posts. Minerva Stomatol. 2009;58:99-106.

6- Asmussen E, Peutzfeldt A, Sahafi A. Finite element analysis of stresses in endodontically treated, dowel-restored teeth. J Prosthet Dent. 2005;94:321-9.

7- Ayad MF, Bahannan SA, Rosenstiel SF. Influence of irrigant, dowel type, and root-reinforcing material on fracture resistance of thinwalled endodontically treated teeth. J Prosthodont. 2011;20:180-9. 8- Bergoli CD, Amaral M, Boaro LC, Braga RR, Valandro LF. Fiber post cementation strategies: effect of mechanical cycling on pushout bond strength and cement polymerization stress. J Adhes Dent. 2012;14:471-8.

9- Bergoli CD, Lima JM, Melo RM, Bottino MA, Valandro LF. Effect of the interfacial area measurement parameters on the pushout strength between fiber post and dentin. Int J Adhes Adhes. 2014;50:7-10.

10- Bortoluzzi EA, Souza EM, Reis JM, Esberard RM, TanomaruFilho $M$. Fracture strength of bovine incisors after intra-radicular treatment with MTA in an experimental immature tooth model. Int Endod J. 2007;40:684-91.

11- Brito-Junior M, Braga NM, Rodrigues DC, Camilo CC, Fariae-Silva AL. Effect of the simulated periodontal ligament on cast post-and-core removal using an ultrasonic device. J Appl Oral Sci. 2010;18:528-32.

12- Camargo $\mathrm{CH}$, Siviero M, Camargo SE, Oliveira SH, Carvalho CA, Valera MC. Topographical, diametral, and quantitative analysis of dentin tubules in the root canals of human and bovine teeth. J Endod. 2007;33:422-6.
13- Clavijo VG, Reis JM, Kabbach W, Silva AL, Oliveira Junior OB, Andrade MF. Fracture strength of flared bovine roots restored with different intraradicular posts. J Appl Oral Sci. 2009;17:574-8.

14- Coelho CS, Biffi JC, Silva GR, Abrahão A, Campos RE, Soares CJ. Finite element analysis of weakened roots restored with composite resin and posts. Dent Mater J. 2009;28:671-8.

15- Erdemir U, Mumcu E, Topcu FT, Yildiz E, Yamanel K, Akyol M. Micro push-out bond strengths of 2 fiber post types luted using different adhesive strategies. Oral Surg Oral Med Oral Pathol Oral Radiol Endod. 2010;110:534-44.

16- Hohmann A, Kober C, Young P, Dorow C, Geiger M, Boryor A, et al. Influence of different modeling strategies for the periodontal ligament on finite element simulation results. Am J Orthod Dentofacial Orthop. 2011;139:775-83.

17- Lanza A, Aversa R, Rengo S, Apicella D, Apicella A. 3D FEA of cemented steel, glass and carbon posts in a maxillary incisor. Dent Mater. 2005; 8:709-15.

18- Marchi GM, Mitsui FH, Cavalcanti AN. Effect of remaining dentine structure and thermal-mechanical aging on the fracture resistance of bovine roots with different post and core systems. Int Endod J. 2008;41:969-76.

19- Mazzoni A, Marchesi G, Cadenaro M, Mazzotti G, Di Lenarda R, Ferrari $M$, et al. Push-out stress for fibre posts luted using different adhesive strategies. Eur J Oral Sci. 2009;117:447-53.

20- McLaren JD, McLaren CI, Yaman P, Bin-Shuwaish MS, Dennison JD, McDonald NJ. The effect of post type and length on the fracture resistance of endodontically treated teeth. J Prosthet Dent. 2009;101:174-81.

21- Mitsui FH, Marchi GM, Pimenta LA, Ferraresi PM. In vitro study of fracture resistance of bovine roots using different intraradicular post systems. Quintessence Int. 2004;35:612-6.

22- Nishimura Y, Tsubota Y, Fukushima S. Influence of cyclic loading on fiber post and composite resin core. Dent Mater J. 2008;27:35661.

23- Palamidakis FD, Panou A, Papadokostaki KG, Leontakianakos G, Stathopoulos VN, Kontakiotis EG. Device and materials for in vitro evaluation of forces developed to teeth and periodontal structures during dental practices. J Dent Biomech. 2013;4:1758736013503648. 24- Pini M, Wiskott HW, Scherrer SS, Botsis J, Belser UC. Mechanical characterization of bovine periodontal ligament. J Periodontal Res. 2002;37:237-44.

25- Rosentritt M, Behr M, Scharnagl P, Handel G, Kolbeck C. Influence of resilient support of abutment teeth on fracture resistance of allceramic fixed partial dentures: an in vitro study. Int J Prosthodont. 2011;24:465-8.

26- Soares CJ, Pizi EC, Fonseca RB, Martins LR. Influence of root embedment material and periodontal ligament simulation on fracture resistance tests. Braz Oral Res. 2005;19:11-6.

27- Soros C, Zinelis S, Lambrianidis T, Palaghias G. Spreader load required for vertical root fracture during lateral compaction ex vivo: evaluation of periodontal simulation and fracture load information. Oral Surg Oral Med Oral Pathol Oral Radiol Endod. 2008;106:e64-70. 28- Sterzenbach G, Kalberlah S, Beuer F, Frankenberger R, Naumann M. In-vitro simulation of tooth mobility for static and dynamic load tests: a pilot study. Acta Odontol Scand. 2011;69:316-8.

29- Wandscher VF, Bergoli CD, Limberger IF, Ardenghi TM, Valandro LF. Preliminary results of the survival and fracture load of roots restored with intracanal posts: weakened vs non-weakened roots. Oper Dent. 2014;39(5):541-56.

30- Wiskott HW, Nicholls JI, Belser UC. Stress fatigue: basic principles and prosthodontic implications. Int J Prosthodont. 1995;8:105-16. 